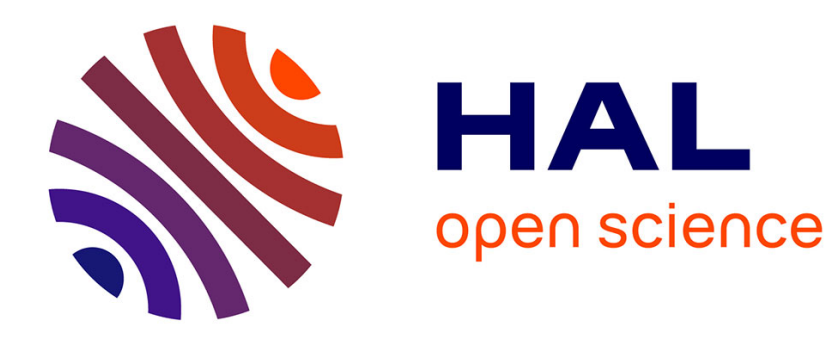

\title{
Momentum expectation values : gradient terms
}

G.I. Plindov, I.K. Dmitrieva

\section{To cite this version:}

G.I. Plindov, I.K. Dmitrieva. Momentum expectation values: gradient terms. Journal de Physique Lettres, 1984, 45 (9), pp.419-425. 10.1051/jphyslet:01984004509041900 . jpa-00232364

\section{HAL Id: jpa-00232364 https://hal.science/jpa-00232364}

Submitted on 1 Jan 1984

HAL is a multi-disciplinary open access archive for the deposit and dissemination of scientific research documents, whether they are published or not. The documents may come from teaching and research institutions in France or abroad, or from public or private research centers.
L'archive ouverte pluridisciplinaire HAL, est destinée au dépôt et à la diffusion de documents scientifiques de niveau recherche, publiés ou non, émanant des établissements d'enseignement et de recherche français ou étrangers, des laboratoires publics ou privés. 
Classification

Physics Abstracts

$31.10-31.20 \mathrm{~L}$

\title{
Momentum expectation values : gradient terms
}

\author{
G. I. Plindov \\ Nuclear Power Engineering Institute, Minsk, U.S.S.R.
}

and I. K. Dmitrieva

Heat and Mass Transfer Institute, Minsk, U.S.S.R.

(Reçu le 28 octobre 1983, révisé le 26 janvier 1984, accepté le 29 février 1984)

\begin{abstract}
Résumé. - Une correction du premier ordre à la valeur moyenne des impulsions $\left\langle p^{b}\right\rangle$, due à l'inhomogénéité de la densité électronique est obtenue dans le cadre de la technique de développement en gradients de Kirzhnits. On obtient une estimation qualitative de $\left\langle p^{b}\right\rangle$ pour les systèmes Coulombiens à plusieurs corps grâce à une transformation d'échelle. Une estimation quantitative de la contribution de l'inhomogénéité aux valeurs moyennes $\left\langle p^{b}\right\rangle$ est obtenue pour l'atome neutre.

Abstract. - The lowest order inhomogeneity correction for the momentum expectation values, $\left\langle p^{b}\right\rangle$, is derived by means of Kirzhnits' gradient expansion technique. The use of the scaling allows a qualitative estimate of $\left\langle p^{b}\right\rangle$ for the Coulomb many-body systems. A quantitative estimate of the inhomogeneity contribution to the expectation values, $\left\langle p^{b}\right\rangle$, for a neutral atom is made.
\end{abstract}

It is known that using the Thomas-Fermi (TF) model the momentum expectation values, $\left\langle p^{b}\right\rangle$, may be expressed through the ones for the single-particle electron density, $n(\mathbf{r})$, of a many-body system :

$$
\left\langle p^{b}\right\rangle=\frac{3}{3+b}\left(3 \pi^{2}\right)^{b / 3}\left\langle n^{\frac{b+3}{3}}(\mathbf{r})\right\rangle \text {. }
$$

The atomic units are used everywhere.

Thus, the calculation of $\left\langle p^{b}\right\rangle$, requiring a knowledge of the density in the momentum space, is replaced by the simpler coordinate integration.

Equation (1) is exact if the electrostatic and exchange interactions are taken into account. This relation is broken when account is taken of contributions of strongly bound electrons, of the inhomogeneity and oscillations of the electron density. Our aim is to obtain corrections for the electron density inhomogeneity and to estimate the main contributions to $\left\langle p^{b}\right\rangle$ for manybody Coulomb systems such as an atom, molecule, etc. 
Let us consider a contribution of the electron density inhomogeneity. For this purpose the gradient expansion technique developed by Kirzhnits [1] is used. To the single-particle approximation, the distribution function for the ground state of a many-body system is expressed as :

$$
f(\hat{r}, \hat{p})=\exp [-i \hat{p} \hat{r}] \theta\left(\varepsilon_{\mathrm{F}}-\hat{T}-V\right) \exp [i \hat{p} \hat{r}]
$$

where $\varepsilon_{\mathrm{F}}$ is the Fermi energy $; \hat{p}, \hat{r}, \hat{T}$ are the operators of momentum, position and kinetic energy; $V$ is the effective single-particle potential; $\theta(x)$ is the Heaviside theta function.

Integrating (2) over momentum, one may obtain the particle number density as a function of the Fermi momentum $p_{\mathrm{F}}^{2}=2\left(\varepsilon_{\mathrm{F}}-V(r)\right)$

$$
n(\mathbf{r})=\frac{1}{4 \pi^{3}} \int_{0}^{p_{\mathrm{F}}} \mathrm{d}^{3} p f(\hat{r}, \hat{p}) .
$$

Equation (2) may be also adopted to determine $\left\langle p^{b}\right\rangle$ as :

$$
\left\langle p^{b}\right\rangle=\frac{1}{4 \pi^{3}} \int \mathrm{d}^{3} r \int_{0}^{p_{\mathrm{F}}} \mathrm{d}^{3} p p^{b} f(\hat{r}, \hat{p}) .
$$

The operators $\hat{p}, \hat{r}, V(r)$ do not commute :

$$
[\hat{p}, V(r)]=-i \nabla V(r) .
$$

Neglect of the non-commutativity of $\hat{p}$ and $V(r)$ in the distribution function $f(\hat{r}, \hat{p})$ yields (1).

The commutator expansion [1] of the arbitrary operator function $F(\hat{a}+\hat{b})$ is used to find gradient expansion terms :

$$
F(\hat{a}+\hat{b})=F(a+\hat{b})-\frac{1}{2} F^{\prime \prime}(a+\hat{b})[\hat{b}, \hat{a}]+\frac{1}{6} F^{\prime \prime \prime}(a+\hat{b})\{[\hat{b}[\hat{b}, \hat{a}]]-[\hat{a}[\hat{b}, \hat{a}]]\}+\cdots .
$$

When the second-order gradients only are preserved, we obtain :

$$
\begin{gathered}
n(\mathbf{r})=\frac{1}{3 \pi^{2}}\left(p_{\mathrm{F}}^{3}-\frac{\left(\nabla p_{\mathrm{F}}^{2}\right)^{2}}{32 p_{\mathrm{F}}^{3}}+\frac{\Delta p_{\mathrm{F}}^{2}}{8 p_{\mathrm{F}}}\right) \\
\left\langle p^{b}\right\rangle=\frac{1}{\pi^{2}}\left\{\frac{\left\langle p_{\mathrm{F}}^{3+b}\right\rangle}{b+3}-\frac{\left(b^{2}-1\right)(b-1)}{96}\left\langle p_{\mathrm{F}}^{b-3} \cdot\left(\nabla p_{\mathrm{F}}^{2}\right)^{2}\right\rangle+\frac{(3-2 b)(b+1)}{72}\left\langle p_{\mathrm{F}}^{b-1} \Delta p_{\mathrm{F}}^{2}\right\rangle\right\}
\end{gathered}
$$

Here $\langle>$ in the RHS means the electron space coordinate averaging. Combining (7) and (6), correct to $\sim[\nabla n(\mathbf{r})]^{2}$ terms, we have :

$$
\left\langle p^{b}\right\rangle=\frac{3\left(3 \pi^{2}\right)^{b / 3}}{b+3}\left\langle n^{\frac{b+3}{3}}(\mathbf{r})\right\rangle+\frac{\left(3 \pi^{2}\right)^{\frac{b-2}{3}}}{216} b\left(b^{2}-3 b+5\right)\left\langle n^{\frac{b-5}{3}}(\mathbf{r})(\nabla n)^{2}\right\rangle .
$$

Green's theorem is used to derive (8). Expression (8) gives the desired estimate of $\left\langle p^{b}\right\rangle$ improved by the effects of the electron density inhomogeneity being taken into account. For $b=2$, the well-known expression [1]

$$
\left\langle p^{2}\right\rangle=2\langle\hat{T}\rangle=\frac{3}{5}\left(3 \pi^{2}\right)^{2 / 3}\left\langle n^{5 / 3}(\mathbf{r})\right\rangle+\frac{1}{36}\left\langle\frac{(\nabla n)^{2}}{n}\right\rangle
$$

is obtained. 
Expression (8) yields the lowest term of the gradient expansion which may be formally written as :

$$
\begin{aligned}
&\left\langle p^{b}\right\rangle=\frac{3\left(3 \pi^{2}\right)^{b / 3}}{b+3} \int \mathrm{d}^{3} r n^{\frac{b+3}{3}}(\mathbf{r})\left\{1+A \frac{(\nabla n)^{2}}{n^{8 / 3}}+\right. \\
&\left.+C\left[\frac{(\nabla n)^{4}}{n^{16 / 3}}+\alpha \frac{\left(\nabla^{2} n\right)^{2}}{n^{10 / 3}}+\gamma \frac{(\nabla n \cdot \nabla)^{2} n}{n^{13 / 3}}+\cdots\right]+\cdots\right\} .
\end{aligned}
$$

One can see an exact structure of expansion (10) for $\left\langle p^{2}\right\rangle$ in [2].

Let us show that this expansion is divergent for the isolated many-electron systems. Consider a system of $N$ electrons in the field of $m$ fixed nuclei with charges $Z_{i}$ located at a distance $R_{i j}$ from each other; assume that $N \leqslant \sum_{i}^{m} Z_{i}$. Bearing in mind that in the outer regions of a system, $n(r) \sim \exp (-a r)$ where $a>0$, it is easy to see that the $l$-th term $\left(l \geqslant \frac{b+3}{2}\right)$ of expansion (10) involves the diverging integral. In particular, only two gradient expansion terms may be used to estimate $\left\langle p^{2}\right\rangle$ and only one term, to find $\langle p\rangle$. At $b<-1$, the gradient expansion is inapplicable, as even the first gradient terms diverge. This disadvantage is due to the assumption that $\left|\nabla n \cdot n^{-4 / 3}(r)\right| \ll 1$, which is inapplicable for the outer regions of a system.

Near the Coulomb centres, the condition $\left|\nabla n \cdot n^{-4 / 3}(r)\right| \ll 1$ is also broken, which results in the necessity to select a contribution of strongly bound electrons. Let us estimate the order of the main contributions to $\left\langle p^{b}\right\rangle$. For this purpose the scaling is employed. The TF scaling

$$
\mathbf{r} \rightarrow Z^{-1 / 3} \mathbf{x} ; \quad n(\mathbf{r}) \rightarrow Z^{2} n(\mathbf{x})
$$

is used to estimate a contribution of the bulk electrons, $\sum_{i}^{m} Z_{i}$ being chosen as $Z$. Substitution of these relations into (1) evidences that the contribution of bulk electrons into $\left\langle p^{b}\right\rangle$ is proportional to $Z^{\frac{2 b+3}{3}}$.

$$
\left\langle p^{b}\right\rangle_{\mathrm{TF}} \simeq Z^{\frac{2 b+3}{3}} f_{1}\left(Z_{1}, \ldots, Z_{m-1} ; R_{1}, \ldots, R_{m}\right)
$$

The behaviour of the electron density near a nucleus, $Z_{1}$, is considered to estimate a contribution of strongly bound electrons. The electron-nucleus cusp condition for a density [3] may be given as :

$$
n(\mathbf{r}) \simeq n(0)\left(1-2 Z_{i} r\right) .
$$

At a small distance from a nucleus $i$ so that $|\mathbf{r}|<\left|R_{i j}\right|_{\min }$ the effect of the remainder nuclei may be neglected to a first approximation. Then, $n(0)$ is mainly determined by the $i$-th nucleus electron density. Recently it has been shown [4] that for an isolated atom with a charge $Z_{i}$

$$
n(0) \simeq Z_{i}^{3}\left(A+B V_{0} Z_{i}^{-2 / 3}\right) \text {. }
$$

Here $A$ and $B$ are constants, and only $V_{0}=\left(\frac{r_{i}}{Z_{i}} V+1\right)_{r_{i} \rightarrow 0}$ depends on the electron-electron interaction. For many-nucleus systems, $A, B$ and $V_{0}$ are functions of $R_{i j}$. Taking into account 
that near the Coulomb centres there exist regions where the electron-electron interaction is small (of the relative order $Z^{-2 / 3}$ ) the contribution of strongly bound electrons may be determined by the scaling of the Schrödinger equation for a system of non-interacting electrons :

$$
\mathbf{r} \rightarrow Z^{-1} \mathbf{y} ; \quad n(\mathbf{r}) \rightarrow Z^{3} n(\mathbf{y}) .
$$

Substitution of (12) into (8) yields the contribution of strongly bound electrons :

$$
\left\langle p^{b}\right\rangle_{\text {s.b. }} \simeq Z^{b} f_{2}\left(Z_{1}, \ldots, Z_{m-1} ; R_{1}, \ldots, R_{m}\right) .
$$

Using the scaling in (10), it is easy to see that all gradient expansion terms must contribute to (13).

Summing the main contributions results in :

$$
\left\langle p^{b}\right\rangle=Z^{\frac{2 b+3}{3}} f_{1}\left(Z_{1}, \ldots, Z_{m-1} ; R_{1}, \ldots, R_{m}\right)+Z^{b} f_{2}\left(Z_{1}, \ldots, Z_{m-1} ; R_{1}, \ldots, R_{m}\right) .
$$

Equation (14) specifies the relative rôle of the main contributions for $Z \gg 1$; it is seen that for $b<3$, the TF contribution is leading, while for $b \geqslant 3$ the strongly bound electron one. As all gradient expansion terms contribute to $\left\langle p^{b}\right\rangle_{\text {s.b. }}$, a conclusion is made that for $b \geqslant 3$, a reliable asymptotic estimate of the expectation value, $\left\langle p^{b}\right\rangle$, may be obtained only if a contribution of the strongly bound electrons is selected.

For an atom this problem, without regard for the gradient terms, was solved [5] using the Scott-Schwinger approach [6].

Now let us show how to estimate a quantitative contribution of the electron density inhomogeneity using the statistical model which correctly treats strongly bound electrons. First of all, note that, apart from an explicit inhomogeneity correction (the second term in RHS of (8)), there is a quantum contribution to the first term of this expression due to gradient corrections for the quasi-classical density. We use the Kompaneets and Pavlovsky (KP) density [7] which includes all first-order corrections with respect to the parameter $\beta=\frac{3}{2}(6 \pi Z)^{2 / 3}$

$$
\rho(x)=\frac{32 Z^{2}}{9 \pi^{3}}\left\{\left(\frac{\psi_{\mathrm{TF}}}{x}\right)^{3 / 2}+\beta\left[\frac{3}{2}\left(\frac{\psi_{\mathrm{TF}}}{x}\right)^{1 / 2} \frac{\psi_{\mathrm{KP}}}{x}+\frac{10}{3} \frac{\psi_{\mathrm{TF}}}{x}-\frac{1}{12}\left(\frac{x}{\psi_{\mathrm{TF}}}\right)^{3 / 2}\left(\frac{\mathrm{d}}{\mathrm{d} x} \frac{\psi_{\mathrm{TF}}}{x}\right)^{2}\right]\right\} .
$$

Here $x=(128 Z)^{1 / 3}\left(9 \pi^{2}\right)^{-1 / 3} r, \psi_{\mathrm{TF}}(x)$ is the TF screening function and $\psi_{\mathrm{KP}}(x)$ is the firstorder quantum correction. Remembering the relation between $\psi_{\mathrm{KP}}(x)$ and the exchange correction, $\psi_{\mathrm{ex}}(x),[8]$

$$
\psi_{\mathrm{KP}}(x)=\frac{1}{3} x^{1 / 2} \psi_{\mathrm{TF}}^{1 / 2}(x)+\frac{11}{9} \psi_{\mathrm{ex}}(x)
$$

it is easy to obtain, from (8), (15) and (16), an explicit expression for $\left\langle p^{b}\right\rangle$ for an atom within the statistical model with regard for corrections for exchange and electron density inhomogeneity

$$
\begin{aligned}
& \left\langle p^{b}\right\rangle=\left\langle p^{b}\right\rangle_{\mathrm{TF}}+\left\langle p^{b}\right\rangle_{\mathrm{ex}}+\left\langle p^{b}\right\rangle_{\mathrm{q}} \\
& \left\langle p^{b}\right\rangle_{\mathrm{TF}}=\frac{3}{3+b}\left(\frac{32}{3 \pi}\right)^{b / 3} Z^{\frac{2 b+3}{3}} \int \psi_{\mathrm{TF}}^{\frac{b+3}{2}}(x) x^{\frac{1-b}{2}} \mathrm{~d} x, \\
& \left\langle p^{b}\right\rangle_{\mathrm{ex}}=\frac{3}{2 \pi}\left(\frac{32}{3 \pi}\right)^{\frac{b-1}{3}} Z^{\frac{2 b+1}{3}}\left[\int \psi_{\mathrm{TF}}^{\frac{b+1}{2}}(x) \psi_{\mathrm{ex}}(x) x^{\frac{1-b}{2}} \mathrm{~d} x+2 \int \psi_{\mathrm{TF}}^{\frac{b+2}{2}}(x) x^{\frac{2-b}{2}} \mathrm{~d} x\right],
\end{aligned}
$$




$$
\begin{aligned}
\left\langle p^{b}\right\rangle_{\mathrm{q}} & =\frac{1}{3 \pi}\left(\frac{32}{3 \pi}\right)^{\frac{b-1}{3}} Z^{\frac{2 b+1}{3}}\left[\int \psi_{\mathrm{TF}}^{\frac{b+1}{2}}(x) \psi_{\mathrm{ex}}(x) x^{\frac{1-b}{2}} \mathrm{~d} x+\right. \\
& \left.+\frac{5}{2} \int \psi_{\mathrm{TF}}^{\frac{b+2}{2}}(x) x^{\frac{2-b}{2}} \mathrm{~d} x+\frac{b^{3}-3 b^{2}+5 b-3}{12} \int\left(\frac{\psi_{\mathrm{TF}}}{x}\right)^{\frac{b-3}{2}}\left(\frac{\mathrm{d}}{\mathrm{d} x} \frac{\psi_{\mathrm{TF}}}{x}\right)^{2} x^{2} \mathrm{~d} x\right] .
\end{aligned}
$$

It should be noted that these expressions are inapplicable in the strongly bound electron region, which is due to the inadequate quasi-classical description. Some terms in (17) contain diverging integrals. For $b \geqslant 3,\left\langle p^{b}\right\rangle_{\mathrm{TF}}$ diverges [5], for $\left.b\right\rangle 1$, the last term in (17d) does. Deleting, according to [9], the contribution for strongly bound electrons once taken into account, one may write :

$$
\begin{array}{r}
\int_{0}^{\infty}\left(\frac{\psi_{\mathrm{TF}}}{x}\right)^{\frac{b-3}{2}}\left(\frac{\mathrm{d}}{\mathrm{d} x} \frac{\psi_{\mathrm{TF}}}{x}\right)^{2} x^{2} \mathrm{~d} x \rightarrow \int_{0}^{x_{\mathrm{m}}}\left[\left(\frac{\psi_{\mathrm{TF}}}{x}\right)^{\frac{b-3}{2}}\left(\frac{\mathrm{d}}{\mathrm{d} x} \frac{\psi_{\mathrm{TF}}}{x}\right)^{2} x^{2}-\left[\frac{1}{x}+\alpha \varepsilon_{\mathrm{S}}\right]^{\frac{b-3}{2}}\right. \\
] \mathrm{d} x+\int_{x_{\mathrm{m}}}^{\infty}\left(\frac{\psi_{\mathrm{TF}}}{x}\right)^{\frac{b-3}{2}}\left(\frac{\mathrm{d}}{\mathrm{d} x} \frac{\psi_{\mathrm{TF}}}{x}\right)^{2} x^{2} \mathrm{~d} x .
\end{array}
$$

Here $\varepsilon_{S}$ is the Fermi energy of strongly bound electrons and $\alpha=\left(9 \pi^{2}\right)^{1 / 3} 2^{-7 / 3} Z^{-4 / 3}$, $x_{\mathrm{m}}=\left(-\alpha \varepsilon_{\mathrm{s}}\right)^{-1}$.

The partial integration of (18) and assumption of $\alpha \varepsilon_{S}=\left.\frac{d \psi_{\mathrm{TF}}}{\mathrm{dx}}\right|_{x=0}$ yield :

$$
\left\langle p^{b}\right\rangle_{\mathrm{q}}^{\prime}=\frac{1}{3 \pi}\left(\frac{32}{3 \pi}\right)^{\frac{b-1}{3}} z^{\frac{2 b+1}{3}}\left[\int_{0}^{\infty} \psi_{\mathrm{TF}}^{\frac{b+1}{2}} \psi_{\mathrm{ex}} x^{\frac{1-b}{2}} \mathrm{~d} x+\frac{12+2 b-b^{2}}{6} \int \psi_{\mathrm{TF}}^{\frac{b+2}{2}} x^{\frac{2-b}{2}} \mathrm{~d} x\right] \text {. }
$$

Thus, the inhomogeneity contribution is of the same order as the exchange one and may be given as

$$
\left\langle p^{b}\right\rangle_{\mathrm{q}}^{\prime}=B_{\mathrm{q}}(b) Z^{\frac{2 b+1}{3}}
$$

The numerical estimate of $B_{\mathrm{q}}(b)$ obtained using the universal functions $\psi_{\mathrm{TF}}(x)$ and $\psi_{\mathrm{ex}}(x)$ has shown that for a neutral atom $\left\langle p^{b}\right\rangle_{\mathrm{q}}^{\prime}$ is a small part of $\left\langle p^{b}\right\rangle_{\mathrm{ex}}$ (Table I).

The operator $p^{4}$ giving the main part of the relativistic correction for atom binding energy requires a special consideration. The corrections for $\left\langle p^{4}\right\rangle$ with regard for the exchange and inhomogeneity, (17c) and (17e), involve diverging contributions (second integrals in RHSs).

Table I. - Values of $B_{\mathrm{q}}(b)$.

\begin{tabular}{llll}
\hline \multicolumn{1}{c}{$b$} & 1 & 2 & 3 \\
\hline$B_{\mathrm{q}}(b)$ & 0.07334 & 0.09814 & 0.2581 \\
\hline$\left\langle p^{b}\right\rangle_{\mathrm{q}}^{\prime} /\left\langle p^{b}\right\rangle_{\mathrm{ex}}$ & 0.2928 & 0.2222 & 0.1456 \\
\hline
\end{tabular}


This is due to the incorrect description of the exchange interaction of strongly bound electrons in the TF model. To improve it is necessary to delete the incorrect TF contribution and to replace it by the correct quantum one which must be obtained by means of the first-order wave function of the $Z^{-1}$ perturbation theory. As these data are absent in the literature, we have adopted a guess based on the fact that $\left\langle p^{3}\right\rangle_{\mathrm{TF}},(17 \mathrm{~b})$, includes the same diverging integral. Expressing

$$
\int \psi_{\mathrm{TF}}^{3}(x) x^{-1} \mathrm{~d} x=\frac{3 \pi}{16} Z^{-3}\left\langle p^{3}\right\rangle_{\mathrm{TF}}
$$

upon replacement of $\left\langle p^{3}\right\rangle_{\mathrm{TF}} \rightarrow\left\langle p^{3}\right\rangle_{\mathrm{TF}}^{\prime}+\left\langle p^{3}\right\rangle_{\text {s.b. }}$ [5], gives :

$$
\begin{gathered}
\left\langle p^{4}\right\rangle_{\mathrm{ex}}=\frac{16}{\pi^{2}} Z^{3} \int_{0}^{\infty} \psi_{\mathrm{TF}}^{5 / 2} \psi_{\mathrm{ex}} x^{-3 / 2} \mathrm{~d} x+\frac{64}{3 \pi^{2}} Z^{3}(\ln Z+0.5578)=\frac{64}{3 \pi^{2}} Z^{3}(\ln Z-0.5657) \\
\left\langle p^{4}\right\rangle_{\mathrm{q}}^{\prime}=\frac{32}{9 \pi^{2}} Z^{3} \int_{0}^{\infty} \psi_{\mathrm{TF}}^{5 / 2} \psi_{\mathrm{ex}} x^{-3 / 2} \mathrm{~d} x+\frac{128}{81 \pi^{2}} Z^{3}(\ln Z+0.5578)= \\
=\frac{128}{81 \pi^{2}} Z^{3}(\ln Z-2.808)
\end{gathered}
$$

Comparison of the numerical values of $\left\langle p^{4}\right\rangle$, using (17), (19) and (20), with the accurate HF data calculated from the Compton profiles [5] has shown that the allowance for the exchange interaction substantially improves the value of $\left\langle p^{4}\right\rangle$ while, already for $Z=36$, the inhomogeneity contribution is about $0.03 \%$ (Table II).

More accurate estimate of $\left\langle p^{4}\right\rangle$ may be obtained using the new statistical theory [10] which allows consistent interpretation of the main quantum effects.

Table II. - Values of $\left\langle p^{4}\right\rangle$ for neutral atoms (expressions (17), (19), (20)).

\begin{tabular}{cclc}
\hline$Z$ & 10 & 18 & 36 \\
\hline$\left\langle p^{4}\right\rangle_{\mathrm{q}}(20)$ & -81 & 77 & 5793 \\
$\left\langle p^{4}\right\rangle_{\mathrm{ex}} \cdot 10^{-3}(19)$ & 1.74 & 29.30 & 3.04 \\
$\left\langle p^{4}\right\rangle_{\mathrm{TF}} \cdot 10^{-5}[5]$ & 0.874 & 10.6 & 192 \\
$\left\langle p^{4}\right\rangle_{10}(17)$ & 0.891 & 10.9 & 195 \\
$\left\langle p^{4}\right\rangle_{\mathrm{HF}} \cdot 10^{-5}$ & 0.983 & 11.4 & 199 \\
\hline
\end{tabular}

\section{Acknowledgments.}

The authors would like to thank Professor J. Schwinger for the copies of the papers which he kindly sent to us before their publication. 


\section{References}

[1] KIRZHnits, D. A., The Methods of the Field Theory in the Many-Body Theory (Gosatomizdat, Moscow) 1963.

[2] Grammaticos, B., Voros, A., Ann. Phys. 123 (1979) 359.

Murthy, D. R., Phys. Rev. A 24 (1981) 1682.

[3] Kato, T., Commun. Pure Appl. Math. 10 (1957) 151.

[4] Dmitrieva, I. K. and Plindov, G. I., J. Physique 43 (1982) 1599.

[5] Dmitrieva, I. K. and Plindov, G. I., J. Physique 44 (1983) 333.

[6] Scott, J. M. C., Philos. Mag. 43 (1952) 589.

Schwinger, J., Phys. Rev. A 22 (1980) 1827.

[7] Kompaneets, A. S. and Pavlovsky, B. S., Zh. Teor. Fiz. 31 (1956) 427.

[8] Dmitrieva, I. K., Plindov, G. I. and Pogrebnya, S. K., J. Physique 43 (1982) 1339.

[9] SChwinger, J., Phys. Rev. A 24 (1981) 2353.

[10] ENGLert, B.-G. and SCHWinger, J. (in press). 